\title{
QUANTUM STATISTICAL DERIVATION OF THE MACROSCOPIC MAXWELL EQUATIONS
}

\author{
by K. SCHRAM \\ Instituut voor theoretische fysica, Rijksuniversiteit, Utrecht
}

\begin{abstract}
Synopsis
The macroscopic Maxwell equations in matter are derived on a quantum statistical hasis from the microscopic equations for the field operators. Both the density operator formalism and the Wigner distribution function method are discussed. By both methods it can be proved that the quantum statistical ensemble averages of the m:croscopic electric and magnetic fields fulfil the usual macroscopic Maxwell equations.
\end{abstract}

\$1. Introduction. The behaviour of slowly varying electromagnetic fields in material bodies is determined by the macroscopic Maxwell equations. Long ago Lorent $z^{1}$ ) derived these equations by applying a kind of averaging procedure to the fundamental equations of his electron theory. More recently Mazur and $\mathrm{Nij}$ boer ${ }^{2}$ ) gave a refined and more general derivation using statistical mechanical ensemble averaging, which avoided certain difficulties in the older treatments. However, all these derivations are essentially based on a classical model of the atom. Since the electrons bound in atoms should be treated quantum mechanically it would be more consistent to give a derivation of the macroscopic Maxwell equations based on quantum theory. Furthermore, since these equations for example serve to define the dielectric constant it would be desirable to put them on a rigorous quantum mechanical basis.

In this paper the macroscopic Maxwell equations are derived from the more fundamental microscopic field equations by means of a quantum statistical averaging procedure. In the following section the basic quantum mechanical equations of motion for a system of charged non-relativistic particles in a quantized electromagnetic field ("system S") will be discussed. In section 3 the quantum statistical ensemble averaging of these equations is carried out with the density operator $\rho$ connected with the system $S$. It should be remarked that a similar method has been applied to a plasma by Brittin ${ }^{3}$ ) to prove that Maxwell's equations are valid for the quantum statistically averaged fields. However, this author only considers the case of a fully ionized gas and does not obtain in this way the general macroscopic equations for matter in an electromagnetic field, including electric and 
magnetic polarization effects. Instead of the density operator Britt in uses the equivalent formalism of the Wigner distribution function (W.d.f.), which has the advantage over the operator technique that it is more closely analogous to the classical formalism. This alternative averaging method will be briefly discussed in the last section of this paper.

$\S 2$. Formulation of the microscopic equations in quantum theory. All classical derivations of the macroscopic Maxwell equations start from the microscopic Maxwell-Lorentz equations for an electromagnetic field $\boldsymbol{e}, \boldsymbol{b}$, which for the case of point charges in rationalized gaussian units can be written as

$$
\begin{gathered}
\operatorname{div} \boldsymbol{b}=0 \\
\dot{\boldsymbol{b}}=-c \operatorname{curl} \boldsymbol{e} \\
\operatorname{div} \boldsymbol{e}=\sum_{k} e_{k} \delta\left(\boldsymbol{R}_{k}-\boldsymbol{R}\right) \\
\dot{\boldsymbol{e}}=c \operatorname{curl} \boldsymbol{b}-\sum_{k} e_{k} \dot{\boldsymbol{R}}_{k} \delta\left(\boldsymbol{R}_{k}-\boldsymbol{R}\right)
\end{gathered}
$$

where $\boldsymbol{R}_{k}$ are the position coordinates of the point clarges $e_{k}$ and $\boldsymbol{R}$ is the radius vector of the field point.

In the Mazur-Nijboer treatment the field strengths $\boldsymbol{e}$ and $\boldsymbol{b}$ are supposed to depend on the coordinates and momenta of the charged particles and also explicilty on space and time, that is, they are defined as functions in the phase space of the particles at one and the same time. Incidentally we remark that it is not immediately obvious that by doing so the retardation is properly taken into account, but this procedure may be justified satisfactorily (cf. Mazur $\left.{ }^{4}\right)$ ).

If one wishes to derive the macroscopic Maxwell equations using quantum statistics it is clear that one should start from some quantum analogue of eqs. (1)-(4).

At first sight one is tempted to treat only the charged particles quantum-mechanically in such a way that they interact via a classical electromagnetic field which is a function in the phase space of the particles. This procedure seems to be justified because one knows that the final result, namely the macroscopic (averaged) equations, do not contain any quantum effect of the field : for example the macroscopic Maxwell equations do not describe the creation and annihilation of photons. Such a semi-classical treatment however cannot be given due to the fact that already in the classical theory a system of charged particles interacting via an e.m. field cannot be described by an exact hamiltonian depending on the canonical variables of the particle only ${ }^{*}$ ).

In the classical derivation of $\mathrm{Mazur}$ and $\mathrm{Nijboer}$ this circumstance did not give rise to difficulties because the hamiltonian did not enter explicitly into their calculations, but in a quantum mechanical version of their theory the use of a hamilton operator seems to be unavoidable. For example, it is not possible to define a current density operator which obeys the equation of continuity without making use of the explicit form of the hamiltonian, as has already been pointed out by Kramers ${ }^{6}$ ).

*) A well known non-relativistic approximation is the Darwin-hamiltonian ${ }^{5}$ ). 
The above mentioned difficulties can be overcome by considering the field as an independent system with an infinite number of degrees of freedom. In the hamiltonian formalism the quantization of the system particles + field in interaction then becomes quite easy. This method of field quantization is very well known and can be found in several textbooks 7). Here only the main results are briefly recalled, using essentially Heitler's notation.

The classical field strengths $\boldsymbol{e}$ and $\boldsymbol{b}$ can be derived from the potentials $\boldsymbol{A}$ and $\phi$ according to

$$
\begin{gathered}
\boldsymbol{e}=-\operatorname{grad} \phi-c^{-1} \dot{\boldsymbol{A}} \\
\boldsymbol{b}=\operatorname{curl} \boldsymbol{A} .
\end{gathered}
$$

These definitions are equivalent to the first two Maxwell equations (1) and (2). Putting

$$
\operatorname{div} \boldsymbol{A}=0 \quad \text { (Coulomb gauge) }
$$

the equations (3) and (4) lead to

$$
\begin{gathered}
-\square A+c^{-1} \operatorname{grad} \phi=c^{-1} \Sigma_{k} e_{k} \dot{\boldsymbol{R}}_{k} \delta\left(\boldsymbol{R}_{k}-\boldsymbol{R}\right) \\
\mathcal{A}=-\Sigma_{k} e_{k} \delta\left(\boldsymbol{R}_{k}-\boldsymbol{R}\right) .
\end{gathered}
$$

The Coulomb gauge will be used throughout because it leads to the simplest method of quantization (only the traverse field has to be quantized); on the other hand it destroys the Lorentz invariant formulation of the theory, but this is of no importance for our purpose. Assuming periodic boundary conditions the vector potential can be expanded into a Fourier series

$$
\boldsymbol{A}=\Sigma_{\lambda}\left\{q_{\lambda}(t) \boldsymbol{A}_{\lambda}+q_{\lambda}{ }^{*}(t) \boldsymbol{A}_{\lambda}{ }^{*}\right\} \quad\left(\operatorname{div} \boldsymbol{A}_{\lambda}=0\right)
$$

where

$$
\boldsymbol{A}_{\lambda}=\sqrt{4 \pi i^{2}} \boldsymbol{a}_{\lambda} \exp i \boldsymbol{k}_{\lambda} \cdot \boldsymbol{r}
$$

and $\boldsymbol{a}_{\lambda}$ represents a unit vector in the polarization direction. Introducing the canonical variables

$$
Q_{\lambda}=q_{\lambda}+q_{\lambda}{ }^{*} \quad P_{\lambda}=-i \nu_{\lambda}\left(q_{\lambda}-q_{\lambda}{ }^{*}\right) \quad\left(\nu_{\lambda}=c\left|\boldsymbol{k}_{\lambda}\right|\right)
$$

the hamiltonian of the transverse field is found to be

$$
H_{\mathrm{tr}}=\Sigma_{\lambda} \frac{1}{2}\left(P_{\lambda}{ }^{2}+\nu_{\lambda}{ }^{2} Q_{\lambda}{ }^{2}\right) \text {. }
$$

The total hamiltonian of the non relativistic particles and the e.m. field in interartion ("system $S$ ") becomes

with

$$
H=\Sigma_{k} \frac{\left(\boldsymbol{p}_{k}-e c^{-1} \boldsymbol{A}_{k}\right)^{2}}{2 m_{k}}+\frac{1}{2} \Sigma_{\lambda}\left(P_{\lambda}^{2}+\nu_{\lambda}^{2} Q_{\lambda}^{2}\right)+\frac{1}{2} \Sigma_{i \neq k} \frac{e_{i} e_{k}}{\left|\boldsymbol{R}_{i}-\boldsymbol{R}_{k}\right|}
$$

$$
\boldsymbol{A}_{k}=\Sigma_{\lambda}\left\{q_{\lambda}(t) \boldsymbol{A}_{\lambda}\left(\boldsymbol{R}_{k}\right)+\text { c.c }\right\} .
$$

For the sake of simplicity the occurrence of a given external field is not taken into account (this may be added easily in the final results); therefore $\boldsymbol{A}$ only depends implicitly on the time through the field variables $q_{\lambda}(t)$.

The theory is then quantized by interpreting $P_{\lambda}, Q_{\lambda}, \boldsymbol{p}_{k}$ and $\boldsymbol{R}_{k}$ as time dependent operators acting on the state vector $|\psi\rangle$ of the system $S$. It turns 
out that the classical relations (5)-(11) formally remain valid as operator equalities. In particular, the field strengths $\boldsymbol{e}$ and $\boldsymbol{b}$ become operators depending on $P_{\lambda}, Q_{\lambda}, \boldsymbol{p}_{k}$ and $\boldsymbol{R}_{\boldsymbol{k}}$ through the definitions (5) and (6). It is easily seen that eqs. (1), (2) and (3) also hold in the quantum theory if the dot on an operator is understood as the time derivative of a Heisenberg operator, e.g.

$$
\dot{\boldsymbol{b}}=i \hbar^{-1}[H, \boldsymbol{b}] \text {. }
$$

Eqs. (4) and (8) have to be modified because the expression $\sum_{k} e_{k} \dot{\boldsymbol{R}}_{k} \delta\left(\boldsymbol{R}_{k}-\boldsymbol{R}\right)$, understood as an operator, is non hermitean since the operators

$$
\dot{\boldsymbol{R}}_{k}=i \hbar^{-1}\left[H, \boldsymbol{R}_{k}\right]=m_{k}^{-1}\left(\boldsymbol{p}_{k}-e c^{-1} \boldsymbol{A}_{k}\right) \text { and } \delta\left(\boldsymbol{R}_{k}-\boldsymbol{R}\right)
$$

do not commute. To obtain a proper current density operator one has to take instead of the classical expression the symmetrized form

$$
\boldsymbol{i}_{\text {op }}=\frac{1}{2} \sum_{k} e_{k}\left\{\dot{\boldsymbol{R}}_{k} \delta\left(\boldsymbol{R}_{k}-\boldsymbol{R}\right)+\delta\left(\boldsymbol{R}_{k}-\boldsymbol{R}\right) \dot{\boldsymbol{R}}_{k}\right\} .
$$

This operator indeed verifies the equation of continuity by virtue of the following theorem $\left.{ }^{6}\right)$ :

If $F\left(\boldsymbol{R}_{\mathbf{1}} \ldots \boldsymbol{R}_{N}\right)$ be an arbitrary function of the position operators $\boldsymbol{R}_{1} \ldots \boldsymbol{R}_{N}$ then

$$
[H, F]=-\frac{1}{2} \sum_{k}\left\{\left[F, \boldsymbol{p}_{k}\right] \cdot \dot{\boldsymbol{R}}_{k}+\dot{\boldsymbol{R}}_{k} \cdot\left[F, \boldsymbol{p}_{k}\right]\right\}
$$

provided $H$, considered as a polynomial in the $p_{k}$, does not contain terms of a degree higher than the second.

With

$$
\left[\boldsymbol{p}_{k}, F\right]=-i \hbar \partial F / \partial \boldsymbol{R}_{k} \text { and } \dot{F}=i h^{-1}[H, F]
$$

this theorem can also be stated as

$$
\dot{F}=\frac{1}{2} \sum_{k}\left\{\frac{\partial F}{\partial \boldsymbol{R}_{k}} \cdot \dot{\boldsymbol{R}}_{k}+\dot{\boldsymbol{R}}_{k} \cdot \frac{\partial F}{\partial \boldsymbol{R}_{k}}\right\}
$$

which is analogous to the classical expression for the total time derivative. Because the hamiltonian (11) has the required form, one finds for the time derivative of the charge density operator

$$
\begin{array}{r}
\frac{\mathrm{d}}{\mathrm{d} t} \sum_{k} e_{k} \delta\left(\boldsymbol{R}_{k}-\boldsymbol{R}\right)=\frac{1}{2} \sum_{k} e_{k}\left\{\frac{\partial}{\partial \boldsymbol{R}_{k}} \delta\left(\boldsymbol{R}_{k}-\boldsymbol{R}\right) \cdot \dot{\boldsymbol{R}}_{k}+\dot{\boldsymbol{R}}_{k} \cdot \frac{\partial}{\partial \boldsymbol{R}_{k}}-\delta\left(\boldsymbol{R}_{k}-\boldsymbol{R}\right)=\right. \\
=-\frac{1}{2} \operatorname{div}_{\boldsymbol{R}} \sum_{k} e_{k}\left\{\delta\left(\boldsymbol{R}_{k}-\boldsymbol{R}\right) \dot{\boldsymbol{R}}_{k}+\dot{\boldsymbol{R}}_{k} \delta\left(\boldsymbol{R}_{k}-\boldsymbol{R}\right)\right\}=-\operatorname{div} \boldsymbol{i}_{\mathrm{op}}
\end{array}
$$

Hence the equation of continuity holds for the charge and current density operators.

To conclude this section the main results may be summarized as follows:

The equations of motions for a system of charged Schrödinger particles 
interacting via a quantized e.m. field $\boldsymbol{e}, \boldsymbol{b}$ are

$$
\begin{gathered}
\operatorname{div} \boldsymbol{b}=0 \\
\dot{\boldsymbol{b}}=-c \operatorname{curl} \boldsymbol{e} \\
\operatorname{div} \boldsymbol{e}=\Sigma_{k} e_{k} \delta\left(\boldsymbol{R}_{k}-\boldsymbol{R}\right) \\
\dot{\boldsymbol{e}}=c \operatorname{curl} \boldsymbol{b}-\frac{1}{2} \sum_{k} e_{k}\left\{\dot{\boldsymbol{R}}_{k} \delta\left(\boldsymbol{R}_{k}-\boldsymbol{R}\right)+\delta\left(\boldsymbol{R}_{k}-\boldsymbol{R}\right) \dot{\boldsymbol{R}}_{k}\right\} .
\end{gathered}
$$

These operator equations are formally identical to the classical Lorentz equations; the field operators $\boldsymbol{e}$ and $\boldsymbol{b}$ depend on all canonical variables of the system $S$ with hamiltonian (11), whereas in the Mazur-Nijboer treatment the classical quantities $\boldsymbol{e}$ and $\boldsymbol{b}$ were considered to be functions in the phase space of the particles only and no exact hamiltonian existed.

\$3. Quantum statistical averaging of the microscopic equations. We now want to consider a system $S$ consisting of $N$ atoms in an e.m.field; following Mazur-Nijboer's notation the atoms will be numbered by the index $k(k=1 \ldots N)$. Each atom $k$ consists of a point nucleus with charge $e_{k}$ and position vector $\boldsymbol{R}_{k}$, and of $Z_{k}$ point electrons with charges $e_{k i}\left(i=1 \ldots Z_{k}\right)$ and positions $\boldsymbol{r}_{k i}$ relative to the $k^{\text {th }}$ nucleus. The momentum operators canonical to $\boldsymbol{R}_{k}$ and $\boldsymbol{r}_{k i}$ will be indicated by $\boldsymbol{P}_{k}$ and $\boldsymbol{p}_{k i}$ respectively. The canonical field variables are denoted by $Q_{\lambda}, P_{\lambda}$ as before. The only difference with the system $S$ from the previous section is that the charged particles are now divided into groups, each consisting of a nucleus with a number of electrons bound to it. It will be clear that for this new system $S$ the basic microscopic equations can be written as

$$
\begin{aligned}
& \operatorname{div} \boldsymbol{b}=0 \\
& \dot{\boldsymbol{b}}=-c \operatorname{curl} \boldsymbol{e} \\
& \operatorname{div} \boldsymbol{e}=\sum_{k} e_{k} \delta\left(\boldsymbol{R}_{k}-\boldsymbol{R}\right)+\sum_{k i} e_{k i} \delta\left(\boldsymbol{R}_{k}+\boldsymbol{r}_{k i}-\boldsymbol{R}\right) \\
& \dot{\boldsymbol{e}}=c \operatorname{curl} \boldsymbol{b}-\frac{1}{2} \sum_{k} \boldsymbol{e}_{k}\left\{\dot{\boldsymbol{R}}_{k} \delta\left(\boldsymbol{R}_{k}-\boldsymbol{R}\right)+\delta\left(\boldsymbol{R}_{k}-\boldsymbol{R}\right) \mid \dot{\boldsymbol{R}}_{k}\right\}- \\
& \left.-\frac{1}{2} \Sigma_{k i} e_{k i}\left(\dot{\boldsymbol{R}}_{k}+\dot{\boldsymbol{r}}_{k i}\right) \delta\left(\boldsymbol{R}_{k}+\boldsymbol{r}_{k i}-\boldsymbol{R}\right)+\delta\left(\boldsymbol{R}_{k}+\boldsymbol{r}_{k i}-\boldsymbol{R}\right)\left(\boldsymbol{R}_{k}+\boldsymbol{r}_{k i}\right)\right\} \text {. }
\end{aligned}
$$

In the classical theory of matter in an e.m. field the macroscopic field strengths are space-time (or ensemble) averages of the corresponding microscopic quantities and it has been shown that these averages fulfil the macroscopic Maxwcll cquations. In the same spirit we want to establish relations between the quantum statistical ensemble averages of the microscopic field operators $\boldsymbol{e}$ and $\boldsymbol{b}$. For this purpose we define the density operator of the system $S$ by

$$
\rho=\sum_{i} p_{i}\left|\psi_{i}\right\rangle\left\langle\psi_{i}\right|
$$

where $\left|\psi_{i}\right\rangle$ represents the state vector of the system $S$ occurring in the ensem- 
ble with a statistical weight $p_{i}$. The average value of an operator $A$ is then obtained from

$$
\langle A\rangle=\operatorname{Tr}(\rho A) .
$$

In the Heisenberg representation $\rho$ is independent of the time; the evolution in time of $\langle A\rangle$ is determined by (cf. ref. 2)

$$
\frac{\partial}{\partial t}\langle A\rangle=\operatorname{Tr}(\rho \dot{A})
$$

where

$$
\dot{A}=i \hbar^{-1}[H, A]+\frac{\partial A}{\partial t} .
$$

Analogous to the classical theory the macroscopic electric field $\boldsymbol{E}$ will now be defined as the ensemble average of the microscopic field operator $\boldsymbol{e}$

$$
\boldsymbol{E} \equiv \operatorname{Tr}(\rho e) .
$$

Similarly the macroscopic magnetic field $\boldsymbol{B}$ is defined by

$$
\boldsymbol{B} \equiv \operatorname{Tr}(\rho \boldsymbol{b}) .
$$

Still using the same notation as Mazur-Nijboer the operators for the atomic electric dipole and quadrupole moment and the magnetic dipole moment are

$$
\begin{aligned}
\boldsymbol{\mu}_{k}^{e l} & =\sum_{i} e_{k i} \boldsymbol{r}_{k i}, \\
\mathrm{q}_{k}{ }^{e l} & =\frac{1}{2} \sum_{i} e_{k i} \boldsymbol{r}_{k i} \boldsymbol{r}_{k i},\left(2^{\text {nd }}\right. \text { rank tensor in dyadic notation) } \\
\boldsymbol{\mu}_{k}{ }^{m} & =\frac{1}{2} c^{-1} \sum_{i} e_{k i} \boldsymbol{r}_{k i} \wedge \boldsymbol{r}_{k i} .
\end{aligned}
$$

The average densities of these moments at the point $\boldsymbol{R}$ are defined as

$$
\begin{gathered}
\boldsymbol{P}(\boldsymbol{R}, t)-\operatorname{Tr}\left\{\rho \sum_{k i} e_{k i} \boldsymbol{r}_{k i} \delta\left(\boldsymbol{R}_{k}-\boldsymbol{R}\right)\right\}, \\
\mathbf{Q}(\boldsymbol{R}, t)=\frac{1}{2} \operatorname{Tr}\left\{\rho \sum_{k i} e_{k i} \boldsymbol{r}_{k i} \boldsymbol{r}_{k i} \delta\left(\boldsymbol{R}_{k}-\boldsymbol{R}\right)\right\}, \\
\boldsymbol{M}(\boldsymbol{R}, t)=\frac{1}{2} c^{-1} \operatorname{Tr}\left\{\rho \sum_{k i} e_{k i} \boldsymbol{r}_{k i} \wedge \dot{\boldsymbol{r}}_{k i} \delta\left(\boldsymbol{R}_{k}-\boldsymbol{R}\right)\right\} .
\end{gathered}
$$

These averages can also be expressed with the aid of the reduced density operator $\rho^{(k)}$ for the $k^{\text {th }}$ atom, whose matrix elements in the coordinate representation are defined as (take for instance $k=1$ )

$$
\begin{gathered}
\left\langle\boldsymbol{R}_{1}{ }^{\prime} \boldsymbol{r}_{1 j^{\prime}}\left|\boldsymbol{\rho}^{(\mathbf{1})}\right| \boldsymbol{R}_{1}{ }^{\prime \prime}, \boldsymbol{r}_{1 j^{\prime \prime}}\right\rangle=\int\left\langle\boldsymbol{R}_{1}{ }^{\prime}, \boldsymbol{R}_{2} \ldots \boldsymbol{R}_{N} ; \boldsymbol{r}_{1 j^{\prime}}, \boldsymbol{r}_{2 j} \ldots \boldsymbol{r}_{N j} ; Q_{\lambda}|\rho|\right. \\
\left|\boldsymbol{R}_{1}{ }^{\prime \prime}, \boldsymbol{R}_{2} \ldots \boldsymbol{R}_{N} ; \boldsymbol{r}_{1 j}{ }^{\prime \prime}, \boldsymbol{r}_{2 j} \ldots \boldsymbol{r}_{N j} ; Q_{\lambda}\right\rangle \mathrm{d} \boldsymbol{R}_{2} \ldots \mathrm{d} \boldsymbol{R}_{N} \\
\Pi_{j} \mathrm{~d} \boldsymbol{r}_{2 j} \ldots \mathrm{d} \boldsymbol{r}_{N j} \Pi_{\lambda} \mathrm{d} Q_{\lambda} .
\end{gathered}
$$

The index $j$ in $\boldsymbol{r}_{k j}$ runs from 1 to $Z_{k}$ and the integration has been carried out over the coordinates of all atoms except $k=1$ and all ficld variablcs $Q_{\lambda}$. Writing (29) in the coordinate representation and performing the integrations over the deltafunctions one finds

$$
\boldsymbol{P}(\boldsymbol{R}, t)=\sum_{k i} e_{k i} \int \boldsymbol{r}_{k i}\left\langle\boldsymbol{R}, \boldsymbol{r}_{k j}\left|\rho^{(k)}\right| \boldsymbol{R}, \boldsymbol{r}_{k j}\right\rangle \prod_{j} \mathrm{~d} \boldsymbol{r}_{k j} .
$$


In a similar way one is able to write $\mathbf{Q}$ and $\boldsymbol{M}$ in terms of the atomic density operator $\rho^{(k)}$. In fact these formulae are quite analogous to the corresponding classical expressions of Mazur-Nijboer since the diagonal elements of the density matrix are the quantum analogue of the classical distribution function.

Rather than rewriting all classical definitions in terms of operators we now turn to the actual ensemble averaging of the equations (19)-(22). Using (24), (25), (27) and (28) one finds very easily starting from (19) and (20)

$$
\begin{gathered}
\operatorname{div} \boldsymbol{B}=0 \\
\frac{\partial \boldsymbol{B}}{\partial t}=-c \operatorname{curl} \boldsymbol{E} .
\end{gathered}
$$

From (21) one obtains in this way

$$
\operatorname{div} \boldsymbol{E}=\operatorname{Tr}\left\{\rho \sum_{k} e_{k} \delta\left(\boldsymbol{R}_{k}-\boldsymbol{R}\right)\right\}+\operatorname{Tr}\left\{\rho \sum_{k i} e_{k i} \delta\left(\boldsymbol{R}_{k}+\boldsymbol{r}_{k i}-\boldsymbol{R}\right)\right\} .
$$

The righthand side of this equation can be evaluated in a way which is perfectly analogous to the classical derivation of Mazur-Nijboer. Using again the reduced (atomic) density operator $\rho^{(k)}$ defined in (32) one has

$$
\begin{aligned}
\operatorname{div} \boldsymbol{E} & =\sum_{k} e_{k} \int\left\langle\boldsymbol{R}, \boldsymbol{r}_{k j}\left|\boldsymbol{\rho}^{(k)}\right| \boldsymbol{R}, \boldsymbol{r}_{k j}\right\rangle \prod_{j} \mathrm{~d} \boldsymbol{r}_{k j}+ \\
& +\sum_{k i} e_{k i} \int\left\langle\boldsymbol{R}-\boldsymbol{r}_{k i}, \boldsymbol{r}_{k j}\left|\rho^{(k)}\right| \boldsymbol{R}-\boldsymbol{r}_{k i}, \boldsymbol{r}_{k j}\right\rangle \Pi_{j} \mathrm{~d} \boldsymbol{r}_{k j} .
\end{aligned}
$$

The expression $\left\langle\boldsymbol{R}-\boldsymbol{r}_{k i}, \boldsymbol{r}_{k j}\left|\rho^{(k)}\right| \boldsymbol{R}-\boldsymbol{r}_{k i}, \boldsymbol{r}_{k j}\right\rangle$ can be expanded in a Taylor series around $\boldsymbol{R}$ if one assumes that as a function of $\boldsymbol{R}-\boldsymbol{r}_{k i}$ it does not change appreciably over distances comparable to the atomic dimensions. One finds after a short calculation, the details of which can be found in ref. 2

$$
\operatorname{div} \boldsymbol{E}=\rho_{0}-\operatorname{div}(\boldsymbol{P}-\operatorname{div} \mathbf{Q})
$$

where $\rho_{0}$ is the (average) true charge density defined by

$$
\begin{aligned}
\rho_{0}=\operatorname{Tr} & \left\{\rho \sum_{k}\left(e_{k}+\sum_{i} e_{k i}\right) \delta\left(\boldsymbol{R}_{k}-\boldsymbol{R}\right)\right\}= \\
& =\sum_{k}\left(e_{k}+\sum_{i} e_{k i}\right) \int\left\langle\boldsymbol{R}, \boldsymbol{r}_{k j}\left|\rho^{(k)}\right| \boldsymbol{R}, \boldsymbol{r}_{k j}\right\rangle \Pi_{j} \mathrm{~d} \boldsymbol{r}_{k j} .
\end{aligned}
$$

Introducing the dielectric displacement

$$
\boldsymbol{D} \equiv \boldsymbol{E}+\boldsymbol{P}-\operatorname{div} \mathbf{Q}
$$

(37) takes the familiar form

$$
\operatorname{div} \boldsymbol{D}=\rho_{0} .
$$

The Maxwell equation (10) has also been derived on the basis of quantum statistics by Jansen ${ }^{8}$ ) using a somewhat different averaging method; this author however starts from the classical microscopic equation (3) and therefore does not seem to develop a consistently quantum-mechanical theory. 
The derivation of the fourth macroscopic Maxwell equation from (22) is again quite analogous to the classical calculation; of course one has to symmetrize products of non-commuting operators like $\dot{\boldsymbol{R}}_{k} \delta\left(\boldsymbol{R}_{k}-\boldsymbol{R}\right)$ etc. in order to obtain real average values, as has been pointed out before. Since this amounts to a mathematically trivial modification it makes no sense to repeat the calculation here. In a notation introduced by Mazur ${ }^{4}$ ) the result can be summarized as follows:

$$
\frac{\partial \boldsymbol{D}}{\partial t}=c \operatorname{curl} \boldsymbol{H}-\boldsymbol{I}
$$

where $\boldsymbol{I}$ is the average true current density defined by

$$
\boldsymbol{I}=\frac{1}{2} \operatorname{Tr}\left[\rho \sum_{k}\left(e_{k}+\sum_{i} e_{k i}\right)\left\{\dot{\boldsymbol{R}}_{k} \delta\left(\boldsymbol{R}_{k}-\boldsymbol{R}\right)+\delta\left(\boldsymbol{R}_{k}-\boldsymbol{R}\right) \dot{\boldsymbol{R}}_{k}\right\}\right]
$$

and $\boldsymbol{H}$ represents the magnetic displacement

$$
\begin{gathered}
\boldsymbol{H}=\boldsymbol{B}-\boldsymbol{M}-c^{-1}\left\{\boldsymbol{P} \wedge \boldsymbol{v}-\left(\nabla_{\boldsymbol{R}} \cdot \mathrm{Q}\right) \wedge \boldsymbol{v}\right\}-\frac{1}{2} c^{-1} \operatorname{Tr}\left[\rho \sum _ { k } \left\{\boldsymbol{\mu}_{k} e l \wedge\left(\dot{\boldsymbol{R}}_{k}\right)-\right.\right. \\
\left.-\left(\nabla_{R} \cdot q_{k}^{e l}\right) \wedge\left(\dot{\boldsymbol{R}}_{k}\right)\right\} \delta\left(\boldsymbol{R}_{k}-\boldsymbol{R}\right)+\rho \sum_{k} \delta\left(\boldsymbol{R}_{k}-\boldsymbol{R}\right)\left\{\boldsymbol{\mu}_{k} e l \wedge\left(\dot{\boldsymbol{R}}_{k}\right)-\right. \\
\left.\left.-\left(\nabla_{R} \cdot q_{k}^{e l}\right) \wedge \dot{\boldsymbol{R}}_{k}\right\}\right]
\end{gathered}
$$

where $v$ is the average velocity of the center of gravity of the system of particles

$$
\boldsymbol{v}=\frac{\operatorname{Tr}\left[\rho \sum_{k} \frac{1}{2} m_{k}\left\{\dot{\boldsymbol{R}}_{k} \delta\left(\boldsymbol{R}_{k}-\boldsymbol{R}\right)+\delta\left(\boldsymbol{R}_{k}-\boldsymbol{R}\right) \dot{\boldsymbol{R}}_{k}\right\}\right]}{\operatorname{Tr}\left\{\rho \sum_{k} m_{k} \delta\left(\boldsymbol{R}_{k}-\boldsymbol{R}\right)\right\}}
$$

and $\left(\dot{\boldsymbol{R}}_{k}\right)$ is an abbreviated notation for the operator $\dot{\boldsymbol{R}}_{\boldsymbol{k}}-\boldsymbol{v}$.

This completes our quantum statistical derivation of the macroscopic Maxwell equations. Though this derivation is very formal it is also quite general: for example, the density operator $\rho$ need not be specified. One might object against the fact that $\rho$ depends on a denumerably infinite set of variables, but this is a common phenomenon in quantum field theory.

Finally we wish to remark that the classical Mazur-Nijboer derivation can be easily reformulated in the infinitely dimensional phase space of the particles + field using the hamiltonian representation of the classical e.m. field. This method might even be preferred to the original formulation since the retarded interaction is taken into account in a fully consistent way. A quantum analogue of the classical derivation in the infinitely dimensional phase space of the system $S$ will be considered in the next section.

§4. Alternative derivation by means of the Wigner distribution function. Instead of carrying out the ensemble averaging with the density operator as has been done in the previous section, one can also make use of the Wigner distribution function (W.d.f.) ${ }^{9}$ ). 
The basic idea of this method is to calculate the quantum mechanical expectation value or, more generally, the quantum statistical ensemble average of an operator by "averaging" the corresponding classical quantity over the phase space with the W.d.f.. Though the W.d.f. is no legitimate probability density in phase space (it may take negative values), it yields the correct quantum statistical average under condition that the quantities to be averaged are related to the corresponding quantum mechanical operators according to Weyl's rule. Accepting this extra postulate one easily performs the transition from classical statistical mechanics to quantum statistics, at least formally, just by replacing the classical d.f. by the W.d.f.. Since we know how to derive the macroscopic Maxwell equations from classical statistical mechanics, it seems attractive to use this method for a quantum statistical derivation.

We will now briefly consider this alternative averaging method, which has also been utilized by Brittin ${ }^{3}$ ) in the case of a plasma, however without mentioning explicitly the validity of Weyl's correspondence rule for this case.

In this derivation one starts from the classical microscopic equations (1)-(4), where $\boldsymbol{e}$ and $\boldsymbol{b}$ should be considered as functions in the infinitely dimensional phase space of the system $S$ with hamiltonian (11) (this means an essential difference with the classical Mazur-Nijboer theory). It is not difficult to prove that the classical fields $\boldsymbol{e}, \boldsymbol{b}$, the charge density

and the current density

$$
\sum e_{k} \delta\left(\boldsymbol{R}_{k}-\boldsymbol{R}\right)
$$

$$
\sum e_{k} \dot{\boldsymbol{R}}_{k} \delta\left(\boldsymbol{R}_{k}-\boldsymbol{R}\right)
$$

respectively correspond to the operators $\boldsymbol{e}, \boldsymbol{b}$,

$$
\sum e_{k} \delta\left(\boldsymbol{R}_{k}-\boldsymbol{R}\right) \text { and } \frac{1}{2} \sum e_{k}\left\{\dot{\boldsymbol{R}}_{k} \delta\left(\boldsymbol{R}_{k}-\boldsymbol{R}\right)+\delta\left(\boldsymbol{R}_{k}-\boldsymbol{R}\right) \dot{\boldsymbol{R}}_{k}\right\}
$$

according to Weyl's rule. For example, from the classical expression

$\sum e_{k} \dot{\boldsymbol{R}}_{k} \delta\left(\boldsymbol{R}_{k}-\boldsymbol{R}\right)=\sum e_{k} m_{k}{ }^{-1} \boldsymbol{p}_{k} \delta\left(\boldsymbol{R}_{k}-\boldsymbol{R}\right)-\sum e_{k}^{2}\left(m_{k} c\right)^{-1} \boldsymbol{A}_{k} \delta\left(\boldsymbol{R}_{k}-\boldsymbol{R}\right)$

one finds by applying Weyl's rule the current density operator

$$
\begin{aligned}
\frac{1}{2} \sum e_{k} m_{k}-1\left\{\boldsymbol{p}_{k} \delta\left(\boldsymbol{R}_{k}-\boldsymbol{R}\right)+\right. & \left.\delta\left(\boldsymbol{R}_{k}-\boldsymbol{R}\right) \boldsymbol{p}_{k}\right\}-\sum e_{k}^{2}\left(m_{k} c\right)^{-1} \boldsymbol{A}_{k} \delta\left(\boldsymbol{R}_{k}-\boldsymbol{R}\right)= \\
& =\frac{1}{2} \sum\left\{\dot{\boldsymbol{R}}_{k} \delta\left(\boldsymbol{R}_{k}-\boldsymbol{R}\right)+\delta\left(\boldsymbol{R}_{k}-\boldsymbol{R}\right) \dot{\boldsymbol{R}}_{k}\right\} .
\end{aligned}
$$

It is important to note that this relation between the classical current density and the current density operator can only be established by using the explicit form of the hamiltonian (11), because we must express each physical quantity as a function of the canonical variables. One now formally introduces the W.d.f. $f_{w}(p, q)$ depending on the canonical variables $\boldsymbol{R}_{k}, \boldsymbol{r}_{k i}$, $\boldsymbol{P}_{k}, \boldsymbol{p}_{k i}$ of the charged particles and $Q_{\lambda}, P_{\lambda}$ of the e.m. field (these variables will be summarized as $p, q$ ) and defines the average electric and magnetic field strength by

$$
\boldsymbol{E} \equiv \int \boldsymbol{e} f_{w} \mathrm{~d} p \mathrm{~d} q \quad \boldsymbol{B} \equiv \int \boldsymbol{b} f_{w} \mathrm{~d} p \mathrm{~d} q
$$


where the notation $\mathrm{d} p \mathrm{~d} q$ indicates that the integration has to be performed over the total phase space of the system $S$. The definitions (45) are equivalent to (27) and (28) by virtue of the validity of Weyl's rule for this case. Further one needs an analogue of the theorem (25) for the time evolution of an ensemble average. If $a(p, q)$ is the classical quantity which according to Weyl's prescription corresponds to the operator A one finds from (25) and (26) by applying some correspondence rules due to Groenewold ${ }^{10}$ )

$$
\frac{\partial}{\partial t}\langle A\rangle=\int f_{w} \frac{\mathrm{D} a}{\mathrm{D} t} \mathrm{~d} p \mathrm{~d} q
$$

with

$$
\frac{\mathrm{D} a}{\mathrm{D} t}=\frac{\partial a}{\partial t}+\frac{2}{\hbar} \sin \frac{\hbar}{2}\left\{\frac{\partial}{\partial p_{H}} \frac{\partial}{\partial q_{a}}-\frac{\partial}{\partial q_{H}} \frac{\partial}{\partial p_{a}}\right\} H(p, q) a(p, q)
$$

where $\partial / \partial p_{I I}$ means $\partial / \partial p$ operating on $H(p, q)$ only. The expression for $\mathrm{D} a / \mathrm{D} t$ is in general not identical to the total time derivative $\mathrm{d} a / \mathrm{d} t$ which occurs in the classical counterpart of (46). In our special case however where the hamiltonian is a polynomial of $2^{\text {nd }}$ degree in the momenta and the quantities to be averaged are not more general than the type

$$
a(p, q)=a_{0}(q)+\sum_{i} a_{i}(q) p_{i}+\sum_{i j} a_{i j} p_{i} p_{j}
$$

one easily verifies by inserting (11) and (48) into (47)

$$
\frac{\mathrm{D} a}{\mathrm{D} t}=\frac{\mathrm{d} a}{\mathrm{~d} t}
$$

Hence (46) may be replaced by

$$
\frac{\partial}{\partial t}\langle A\rangle=\int \frac{\mathrm{d} a}{\mathrm{~d} t} f_{w} \mathrm{~d} p \mathrm{~d} q
$$

which is formally the same as the corresponding classical property. As has already been mentioned it is not difficult to reformulate the classical Mazur-Nijboer derivation in the infinitely dimensional phase space of the system $S$. The field strengths $\boldsymbol{e}$ and $\boldsymbol{b}$ and also the distribution function then become functions of all field and particle variables, instead of functions of the particle variables only. Except for this change in interpretation, all formulae of Mazur-Nijboer remain the same. By virtue of the important theorem (50) this classical averaging procedure can be easily transformed into a quantum statistical derivation if one only replaces the classical distribution function by the W.d.f.. The reduced d.f. becomes the reduced W.d.f., which can be proved to correspond according to Weyl's rule to the reduced density operator. Evidently this derivation goes completely parallel to the operator treatment presented in the previous section; it is obtained from the latter by applying Weyl's correspondence rule. 
Acknowledgement. The author is greatly indebted to Professor B. R. A. Nijboer, who suggested the present investigation, for his valuable advice and stimulating criticism throughout this work.

Received 19-8-60.

REFERENCES

1) Lorent $\%$, H. A., linc. Math. Wiss. V 2, Heft 1, S.200.

2) Mazur, P. and Nijboer, B. R. A., Physica 19 (1953) 971.

3) Brittin, W. E., Phys. Rev. 106 (1957) 843.

4) Mazur, P., Advances Chem. Phys. I, 310, New York 1958.

5) Darwin, C. G., Phil. Mag. 39 (1920) 537.

6) Kramers, H. A., Hand- und Jahrb. Chenl. Phys. I, \$ 44, Leipzig 1938.

7) See c.g. Heitler, W., The Quantum Theory of Radiation, 3rd ed. Ch. II, Oxford 1954.

8) Jansen, L.., Phys. Rev. 112 (1958) 434.

9) Wigner, l., Phys. Rev. 40 (1932) 749. See also ref. 10.

10) Groenewold, H. J., Physica 12 (1946) 405.

Schram, K., and Nijboer, B. R. A., Physica 25 (1959) 733.

\title{
ERRATUM
}

\section{A group theoretical proof of Kramers' theorem}

\author{
[Physica 26 (1960) 61]
}

by Paul H. E. Meijer

The method used to show that odd-electron representations are always of the second kind is incorrect. The criterion of Frobenius and Schur is valid only for irreducible representations. In the case of odd numbers of electrons all irreducible components of the reducible representation happen to be of the same kind. Under these special circumstances the criterion is fulfilled, even for the reducible case. For an independent proof along these lines one would have to show first that all representations are indeed of the same kind, and since I do not see an independent way to do this, I would prefer to retract the proof altogether.

I would like to thank Mr. H. V. McIntosh for pointing out this error to me. 